\title{
Medicare Value-Based Purchasing: Non-Payment for Selected Hospital-Acquired Conditions
}

T. Valuck

Qualitätsmanagement

Schlüsselwörter

- Medicare

Pay for performance

$\checkmark$ Qualität

Komplikationen

Key words

Medicare

pay-for-performance

quality

complications
Institut

Centers for Medicare \& Medicaid Services, Baltimore, USA

\section{Bibliografie}

Dol $10.1055 / \mathrm{s}-0028-1085587$ Dtsch Med Wochenschr 2008; 133: S140-S143 - (c) Georg Thieme Verlag KG Stuttgart . New York · ISSN 0012-0472

\section{Korrespondenz}

Thomas B. Valuck, MD, JD

Medical Officer \& Senior Adviser Center for Medicare

Management

Centers for Medicare \&

Medicaid Services

Mail Stop C5-15-02,

7500 Security Boulevard

Baltimore, MD 21244

Tel. +01 (410) 786-7479

eMail thomas.valuck@

cms.hhs.gov

\section{Introduction}

The Centers for Medicare \& Medicaid Services (CMS) is transforming the Medicare program from passive payer to active purchaser of higher quality more efficient health care for Medicare beneficiaries through value-based purchasing (VBP). VBP connects payment to performance, and is often referred to as „pay-for-performance“ or „P4P.“ VBP initiatives use measurement data for payment incentives, but also for public reporting, which provides a strong non-financial incentive for performance improvement. Measurement results are publicly reported on a suite of „Medicare Compare“ websites covering hospitals, nursing homes, home health agencies, and dialysis facilities.

CMS has launched Medicare VBP initiatives, which are in various stages of implementation, across multiple settings of care. This article will address Medicare background and VBP generally, including VBP principles and demonstration projects, but will focus specifically on the Medicare hospital-acquired conditions payment provision and the associated present on admission indicator reporting.

\section{Medicare Background}

Medicare is the public health insurance program for American senior citizens (age 65 or older), some disabled people under age 65 , and all people with end-stage renal disease. In 2007, there were 44 million Medicare beneficiaries, of which $84 \%$ were age 65 or older.

In 2007, Medicare spending accounted for $\$ 430$ billion or $15 \%$ of the annual federal budget. This amounts to $20 \%$ of the nation's total health care spending. Total health care spending is $16 \%$ of the nation's gross domestic product (GDP), so Medicare spending is 3-4\% of GDP. Spending per beneficiary now tops $\$ 6,000$ per year. Medicare currently spends about $\$ 130$ billion annually for hospital inpatient services. Under the funding and spending requirements in current statute, Medicare is projected to grow by an average rate of $7 \%$ per year over the next 10 years and become insolvent by 2019, as more beneficiaries will be supported by fewer taxpaying workers over time.
CMS manages the Medicare program, as well as the Medicaid and State Children's Health Insurance Program. Medicaid and SCHIP are statebased public health insurance programs that are jointly funded by the federal and state governments.

\section{Medicare Value-Based Purchasing}

The Medicare Quality Improvement Roadmap, one of CMS' strategic planning documents, defines the Medicare program's vision for quality as, „The right care for every person, every time.“ Under the Roadmap, quality is construed broadly to include all of the six key dimensions of health care quality identified by the Institute of Medicine: safety, effectiveness, efficiency, patient centeredness, timeliness, and equitability. Valuebased purchasing is one of the five strategies in the quality improvement roadmap; others include: (1) working through public and public-private partnerships; (2) measuring quality and reporting comparative results, (3) encouraging the adoption of effective health information technology, and (4) promoting innovation and the evidence base for the effective use of technology.

Medicare program statutory authorities give CMS numerous VBP tools. The Agency applies measurement, payment incentives, public reporting, conditions of participation, coverage policy, and the Quality Improvement Organizations to VBP initiatives. VBP initiatives take various forms, such as pay-for-reporting, pay-for-performance, gainsharing, competitive bidding, bundled payment, coverage decisions, and direct provider support.

VBP is meant to shift from quantity-based to quality-based payment by responding to opportunities for quality improvement and for avoiding unnecessary costs in the provision of care. The quality improvement opportunity is welldocumented in the literature, as is wasteful spending on overuse, misuse, and errors. As an example, researchers at Dartmouth University have documented five-fold variations in Medicare spending for certain procedures and services across the country, and Medicare data shows an inverse relationship between the cost and the quality of care.

There is broad and deep support for Medicare VBP from the Administration and from Congress. Congress has included VBP authorities in some 
form in each of its Medicare Acts of this decade. The Institute of Medicine and the Medicare Payment Advisory Commission, advisers to Congress and the Administration on Medicare policy, have strongly supported Medicare VBP. In addition, VBP is increasingly being encouraged by employer coalitions and used by private sector payers to increase the value of the health care services they are purchasing.

\section{Medicare VBP Demonstrations}

CMS has numerous demonstration and pilot projects underway to study the application of VBP tools to Medicare payment systems. These projects span settings from hospitals to physician practices to nursing homes to home health agencies. The projects are testing performance-based payment through various approaches, including pay-for-performance, gainsharing, bundled payment, care coordination, disease management, and use of health information technology.

In the hospital inpatient setting, the Premier Hospital Quality Incentive Demonstration (HQID) has shown positive results under a performance-based payment model. The HQID rewarded top decile performers on a set of 35 quality indicators in five clinical areas with a $2 \%$ bonus, while second decile performers were awarded a $1 \%$ bonus. The incentives resulted in aggregate quarter-over-quarter improvement at the median for all clinical areas for the 250 participating hospitals. The five clinical areas measured were acute myocardial infarction, coronary artery bypass graft surgery, heart failure, hip and knee replacement procedures, and pneumonia.

In the physician practice setting, the Physician Group Practice Demonstration (PGP Demo) has shown early positive results under a shared savings and performance-based payment model. Under the PGP Demo, the 10 large (greater than 200 physicians) group practices are eligible to earn up to $80 \%$ of the aggregate savings that they generate for their Medicare patients by achieving performance benchmarks on a set of quality measures. The 32 quality measures address the care delivered to patients with heart failure, coronary artery disease, and diabetes. The groups have improved performance by redesigning clinical care processes and investing in health information technology to better identify and act on gaps in care.

\section{Medicare Hospital-Acquired Conditions Payment Provision}

CMS has a number of ongoing Medicare VBP initiatives for hospitals beyond the HQID project, including hospital pay-forreporting on 40 quality measures and the submission of a plan for hospital pay-forperformance to Congress. In addition, CMS' implementation of the Medicare hospital-acquired conditions (HACs) payment provision and the associated present on admission (POA) indicator reporting has garnered a great deal of attention from the media and strong reactions from stakeholders.

\section{A. HAC Background}

In 1999, the Institute of Medicine estimated that as many as 98,000 American die each year as a result of medical errors and estimated the total cost of these errors to be \$17-29 billion per year. In 2000, the Centers for Disease Control and Prevention (CDC) estimated that hospital-acquired infections add nearly $\$ 5$ billion to US health care costs annually. Moreover, a 2007 Leapfrog Group survey of 1,256 hospitals found that $87 \%$ of those hospitals do not consistently follow recommendations to prevent many of the most common hospital-acquired infections.

\section{B. HAC \& POA Statutory Authority}

As one approach to combating preventable HACs, including infections, Congress required CMS to begin adjusting Medicare hospital payments to encourage the prevention of certain conditions. Specifically, Section 5001(c) of the Deficit Reduction Act of 2005 requires CMS to select conditions that will no longer trigger higher payment for complications when those conditions arise during hospitalization. Beginning October 1, 2008 , cases with HACs will be paid as though the selected complicating conditions were not present; that is, Medicare will only pay the basic rate for uncomplicated care.

The selected complicating conditions must meet the following statutory criteria: (1) be high cost, high volume, or both; (2) trigger higher payment when present as a complication; and (3) be considered reasonably preventable through the application of evidence-based prevention guidelines. The list of conditions can be revised from time to time, as long as it contains at least two conditions.

The statute also authorized CMS to collect a new data element specifying whether diagnoses, including complications, on Medicare claims were present on admission. On October 1, 2007, CMS began requiring hospitals to submit a POA indicator for every diagnosis on a claim to identify which conditions were acquired during hospitalization.

\section{HAC Payment Incentives}

The Medicare inpatient perspective payment system (IPPS) generally encourages hospitals to treat patients efficiently. Hospitals receive the same diagnosis-related group (DRG) payment, regardless of the patient's length of stay in the hospital or the intensity of the services provided. Thus, hospitals have an incentive under the IPPS to avoid unnecessary costs in the delivery of care.

However, complications such as infections, can generate higher Medicare payments in two ways. First, the treatment of complications can increase the cost of a hospital stay enough to generate an outlier payment. Nonetheless, outlier payment methodology requires that a hospital experience a large loss on an outlier, which serves as an incentive for hospitals to prevent outliers.

Second, complications can generate higher Medicare payments based on the presence of a complicating condition (CC) or major complicating condition (MCC). For example, the uncomplicated stroke DRG (066) pays $\$ 5,350$, while the stroke with CC DRG (065) pays $\$ 6,200$ and the stroke with MCC DRG (064) pays $\$ 8,050$. If a complication is on the CC or MCC list, then the hospital receives a higher DRG payment, except when selected HACs are the only complications on a claim. Medicare will continue to assign a case to a higher paying DRG if the selected condition is present on admission or if any non-selected complication is present on the claim. This means that the HAC payment provision will often not apply when HACs are present on the claim, as it 
is typical for Medicare beneficiaries to have several complications and the list of selected HACs is relatively short.

\section{HAC Selection}

CMS clinical quality experts worked closely with public health and infectious disease professionals from the CDC to identify candidate preventable HACs, review public input, and select HACs. CMS and CDC staff also collaborated on the process for hospitals to submit the POA indicator for each diagnosis on Medicare claims and on setting the payment implications for the various POA reporting options. HACs were selected through formal notice-and-comment rulemaking published in the Federal Register, as required by the Administrative Procedures Act to maximize public input into public policymaking.

To determine candidate conditions, CMS and CDC staff evaluated each condition against the DRA criteria. These criteria severely limit the universe of options. The first statutory criterion requires that a selected condition be important to the Medicare program and its beneficiaries. Medicare data must support that the selected condition is high cost, high volume, or both. The POA indicator has only recently begun to be collected for Medicare claims, which hindered the analysis of potential conditions.

The second statutory criterion requires that a selected condition trigger higher Medicare payment. To do so, a condition must be represented by a diagnosis code that clearly identifies that condition, is designed as a CC or an MCC (as explained above), and results in the assignment of the case to a higher paying DRG. This is, a selected condition must be a CC or MCC diagnosis code that would, in the absence of the HAC payment provision, result in the assignment of a higher paying DRG.

The third statutory criterion requires that a selected condition have evidence-based guidelines for its prevention. A number of entities develop and disseminate these guidelines, including the Healthcare Infection Control Practices Advisory Committee (HICPAC), professional organizations, and academic institutions. The absence of prevention guidelines for many potential candidate conditions, including infectious conditions, limits the universe of candidate conditions.
Another aspect of the third statutory criterion requires that a selected condition be considered reasonably preventable when the interventions in the guidelines are followed. The absence of evidence quantifying the extent to which the application of evidence-based guidelines results in the prevention of potential candidate conditions limits the universe of candidate conditions.

After considering public comments received through formal rulemaking, CMS and CDC experts identified ten categories of conditions that will have payment implications under the HAC provision beginning October 1, 2008.

1. Foreign object unintentionally retained after surgery

2. Air embolism

3. Blood incompatibility

4. Pressure ulcer stages III \& IV

5. Falls and trauma

\section{Fracture}

Dislocation

Intracranial injury

Crushing injury

Burn

Electric shock

6. Catheter-associated urinary tract infection

7. Vascular catheter-associated infection

8. Manifestations of poor glycemic control

9. Surgical site infection

Mediastinitis following coronary artery bypass graft surgery

Following certain orthopedic procedures

Following bariatric surgery for obesity 10.Deep vein thrombosis or pulmonary embolism following total knee and hip replacement procedures

\section{E. POA Reporting}

A POA indicator is necessary to identify which conditions were acquired during hospitalization for Medicare payment purposes, as well as being valuable for broader public health uses of Medicare data. There are five POA indicator reporting options established by the National Uniform Billing Committee: (1) the „Y“ reporting option indicates that the condition was present on admission, (2) the „W“ reporting option affirms that the provider has determined based on data and clinical judgment that it is not possible to document when the onset of the condition occurred, (3) the „N" reporting option indicates that the condition was not present on admission, (4) the „U“ re- porting option indicates that the documentation is insufficient to determine if the condition was present on admission, and (5) the „1“ reporting option signifies exemption from POA reporting.

Under the Medicare HAC payment provision, CMS will pay the higher DRG for diagnoses coded „Y“ or „W,“ but will not pay the higher DRG for diagnoses coded „N“ or „U.“ The „1“ reporting option is rarely used for Medicare claims. While the „W" affirms that a determination was made based on clinical evidence that it is not possible to document the onset of the condition, the „U“ simply reflects insufficient documentation. It is expected that not paying more for diagnoses coded with „U“ indicators will encourage better medical record documentation.

Collection of the POA indicator will also provide important information for enhancing public health. Researchers can use POA data for risk adjusting quality measurement data and to gain insights into the incidence of conditions in the community and in hospitals. The POA data can be analyzed for only Medicare beneficiaries or can be combined with private sector or state POA data to support broader conclusions. In addition, POA data could inform publicly reported information to support better health care decision making by consumers and professionals.

\section{F. HAC Provision Enhancements and} Future Issues

Each year through IPPS rulemaking, CMS will consider refinements to the HAC list and potential candidate conditions to add to the list. This might include the consideration of additional categories of conditions, the expansion of existing categories, and the reconsideration of conditions that have previously been proposed but not selected. The ability to select additional conditions will depend on the development of evidence-based prevention guidelines such that when those guidelines are followed, the conditions can be considered reasonably preventable. In addition, having the POA indicator as part of the Medicare claims data will facilitate identification of candidate HACs.

Several means to make the HAC payment policy more precise could be considered in the future, including risk adjustment, application of a more sophisticated pay- 
for-performance model, and the adoption of ICD-10. Rather than not paying any additional amount when a selected HAC occurs during hospitalization, payment reductions could be made proportional to the patient's or patient population's risk - the relative likelihood of acquiring a particular condition during hospitalization. This approach would recognize that medical history, co-morbidities, and severity of illness, among other factors, affect the expected occurrence of complications. The application of a pay-for-performance model based on the measured occurrence of rates of conditions would be a more meaningful, actionable, and fair way to adjust a hospital's payments up or down based on the incidence of HACs in that hospital over time. The United States currently uses ICD-9, but the adoption of ICD-10 would provide a more robust infrastructure for accurately identifying HACs.

\section{Conclusion}

$\nabla$

CMS is applying a range of Medicare statutory authorities to promote higher quality and efficiency through VBP. CMS is experimenting with the use of payment incentives through various demonstration projects, while proceeding with implementation of performancebased payment for some care settings. For hospitals, CMS is implementing a provision under which hospitals will no longer be paid more for selected complicating conditions that arise during hospitalization, thereby encouraging hospitals to prevent the occurrence of those complications. To determine which conditions are hospital-acquired, CMS has begun collecting a present on admission indicator that will also provide valuable information for enhancing public health. CMS' use of VBP payment incentives is enhancing the value of the Medicare dollar.
Author's declaration: The author has no conflicts of interest to disclose. 